\title{
Repenser Le Rôle Des Institutions De Conservation De La Nature À L'ère De La Globalisation En Afrique: À Propos Du Parc National De La Salonga (RDC)
}

\author{
Mampeta Wabasa Salomon \\ $\mathrm{PhD}$, Sociologue Environnementaliste \\ Université de Kisangani, République Démocratique du Congo
}

doi: 10.19044/esj.2017.v13n11p92 URL:http://dx.doi.org/10.19044/esj.2017.v13n11p92

\begin{abstract}
In the Democratic Republic of the Congo (DRC), the protectionist conservatism influenced by colonialism, which exploited African countries for the prosperity of the colonizing countries, still has a high visibility in the Salonga National Park (PNS). If, in theory, the Central Africans seem to free themselves from the colonial powers on their land, in practice they are still there. The hostility of settlers who have become neo-colonists to the development of Central Africa remains intact, he adds (Ndinga, 2003). This reflects a "logic from above" that has disregarded local values. Yet, in the era of sustainable development and globalization, African protected areas appear to be essential tools for States to reposition themselves in a complex set of actors with the aim of capturing and using the new environmental rent (Giraut, Guyot, \& Houssay-Holzschuch, 2003). This is a "bottom-up logic", placing people at the heart of all activities and aiming to reorganize their long-term relationships with the environment. From these two logics, a third "logic from the other side" emerges, reflecting a collective awareness of the fragility of the planet. The restoration of the rights of Africans in the various national frameworks constitutes a major challenge for the contemporary management of African protected areas. Because the protected areas inherited from the different colonial systems must accompany the change in management methods and the redefinition of their functions in order to better serve the local community in the long-term.
\end{abstract}

Keywords: Protectionist Conservationism, Globalization, Community Conservation, Salonga, Democratic Republic of the Congo 


\section{Résumé}

En République Démocratique du Congo (RDC), le conservationnisme protectionniste influencé par le colonialisme, qui exploitait les pays africains pour la prospérité des pays colonisateurs, a encore une grande visibilité au Parc National de la Salonga (PNS). Si, en théorie, les Africains du centre semblent s’être libérés de puissances coloniales sur leurs terres, en pratique, elles sont toujours là. L'hostilité des colons devenus des néo-colons au développement de l'Afrique centrale reste intacte (Ndinga, 2003). Ceci traduit une «logique d'en haut » qui a méconnu les valeurs locales. Et pourtant, à l'heure du développement durable et de la globalisation, les aires protégées africaines apparaissent pour les Etats comme des outils essentiels de repositionnement dans un jeu d'acteurs complexe avec pour enjeu la captation et l'utilisation de la nouvelle rente environnementale (Giraut, Guyot et Houssay-Holzschuch, 2003). Il s’agit là d'une « logique d'en bas », qui replace l’homme au cœur de toute activité et qui vise à réorganiser ses relations de long terme avec l'environnement. De ces deux logiques, il en découle une troisième "la logique d'en face ", qui traduit une prise de conscience collective de la fragilité de la planète. La restauration des droits des Africains dans les différents cadres nationaux constitue un enjeu majeur pour la gestion contemporaine des aires protégées africaines. Car les aires protégées héritières de différents dispositifs coloniaux, doivent accompagner la mutation des modes de gestion et la redéfinition de leurs fonctions afin de mieux servir durablement le local.

Mots clés : Conservationnisme protectionniste, globalisation, conservation communautaire, Salonga, République Démocratique du Congo

\section{Introduction}

De par son étendue, le Parc National de la Salonga (PNS), en République Démocratique du Congo (RDC), offre l'image d'un minicontinent. Ses réalités socio-économiques, écologiques, politiques et même culturelles d'une grande diversité font que ce vaste parc demeure à la fois connu et méconnu de ses fils. Cette complexité tient à son histoire, à sa position géographique, à son inaccessibilité et surtout à sa superficie (36.000 $\mathrm{Km}^{2}$ ) qui est plus étendue que celle de la Belgique” (Sournia, 1998). Tout cela est source de déboires socio-économiques et même culturels pour les populations riveraines, prises entre leur impérieux besoin de survie et la rigidité des mesures de conservation du PNS dont elles dépendent.

Les disparités constatées pendant toutes les missions à la Salonga, entre des communautés très pauvres et ce riche patrimoine dit mondial ne peuvent laisser les chercheurs indifférents. En effet, il est évident que cet état de choses ne peut manquer des conséquences sur le devenir dudit parc, à 
l'aube de ce troisième millénaire, où toutes les nations du monde se préoccupent d'améliorer leurs conditions d'existence et de parvenir ainsi à une gestion rationnelle des ressources naturelles, gage d'un développement durable.

Dans ce contexte, l’objectif poursuivi dans le présent papier est d'examiner, en cette période de globalisation, les causes du blocage persistant dans les rapports sociaux entre les acteurs de la conservation (l'Etat, la société civile et les ONG, d'une part, et les communautés locales, de l'autre) au Parc National de la Salonga. Autrement dit, pourquoi la conservation communautaire n'est-elle pas effective à la Salonga ? Au-delà de cette question fondamentale, il est pertinent de savoir aussi ce qu'il faut faire pour résoudre ce problème observé. L’hypothèse principale dans cette étude est que l'inadéquation entre les mesures de conservation et le vécu des communautés riveraines entraîne le blocage des rapports entre les acteursclés pour l'émergence d'une conservation communautaire durable au PNS. Conscient de cette persistance du problème s'inscrivant dans une longue durée, nous pensons que repenser les normes et les institutions de conservation est une piste prometteuse pour une conservation communautaire effective et, par voie de conséquence, un développement durable des communautés riveraines à la Salonga.

Concernant la posture théorique adoptée, signalons que notre attitude consiste, non pas à considérer les opinions et idées des communautés locales comme d'un intérêt secondaire, mais bien plutôt à revaloriser leurs discours et représentations, et à attacher plus d'importance aux savoirs locaux au lieu d’imposer un modèle de progrès et de connaissance basés sur l'expérience occidentale. Dans cette dernière vision, nous risquerions de sacrifier des valeurs au profit des modèles. A ce sujet, Bertin et al (2003) pensent que le développement local est ainsi une pratique institutionnalisée et instrumentalisée par les politiques surplombantes, européennes, étatiques et régionales. Cette logique répond et fait écho, souvent, aux tendances d'une pensée scientiste faisant disparaître les lois au profit de modèles. En fait, nous prônons une réappropriation des normes et des institutions de conservation de la nature, une manière de les repenser localement au regard de la réalité socio-culturelle de l'Africain en général et du Congolais en particulier. Ainsi que le notent encore Bertin et al, «Les conceptions géographiques et territoriales du développement pris dans leurs réalités intrinsèques, à leur identité, aux traits particuliers ${ }^{43}$ des communautés et les conceptions sectorielles qui l'alignent sur les modèles de la technostructure, soumis aux impératifs de l’institué, de la verticalité. » (2006).

\footnotetext{
${ }^{43}$ Nous employons à dessein cette notion dans le sens que lui donne René Lourau : « La signification particulière, dynamique de l'institution se réfère à l'acte d'instituer, de fonder, de modifier le système » in L'analyse institutionnelle, Paris, Ed de Minuit, 1973, p. 35.
} 


\section{Méthodologie}

Notre propos méthodologique couvre, à la fois, la procédure de récolte de données, le rappel du cadrage théorique pour l’interprétation de faits observés et la présentation du terrain d’enquêtes. En 2004, grâce à une série d'enquêtes socio-économiques (enquêtes de terrain), nous nous sommes rendu au PNS - d'abord pendant six mois, ensuite pendant neuf mois en 2006 - sous la houlette de Wildlife Conservation Society (WCS en sigle), une organisation internationale chargée de conservation de la nature, opérant en RDC. Nous avons alors observé, en qualité de chercheur, des localités où des populations vivent misérablement, perdues au fond de la forêt équatoriale, tout en étant à proximité du PNS, bien identifié comme un patrimoine mondial de l'UNESCO. Nous avons également constaté, pendant cette période, l'indifférence, voire la méfiance entre la population et les agents du parc. En 2014, nous avons été recruté par la même organisation, cette fois-ci, pour réaliser une étude socioéconomique et un recensement, d'abord sur la communauté Yaelima vivant dans le sud du PNS (Novembredécembre 2014), ensuite sur les kitawalistes vivant dans le Nord dudit parc (avril-mai 2015). Ce dernier groupe comporte de nombreuses traces historiques d'existence dans la plupart des provinces de l'Est de la RDC à la suite des vagues de relégations connues sous la période coloniale (Amuri, 2012).

Au-delà de ces brèves considérations purement méthodologiques, il est fondamental de faire mention du processus de théorisation de faits observés. En d’autres termes, nous avons le devoir d’éclairer le processus de compréhension sociologique du blocage qui s’observe au niveau des rapports sociaux entre les acteurs en présence à la Salonga, en vue d'atteindre une conservation citoyenne, donc durable. Pour ce faire, deux principaux paradigmes ont été retenus, en raison de leur capacité heuristique pour rendre compte de l'état de conservation de la nature et du vécu des populations riveraines au PNS.

En effet, le premier paradigme, appelé “ logique d’en haut”, consacre la dépendance et la persistance des structures capitalistes de domination et d'exploitation en matière de conservation de la nature qui se trouve encore cristallisée au sein des institutions concernées. L’Afrique toute entière, et particulièrement la RDC, demeure dépendante de l'Occident en matière de conservation de la nature. Que les normes conçues pour les parcs occidentaux soient appliquées aux parcs africains et congolais, cela pose un problème de cohérence évident. Beaucoup d'activités de ce secteur sont financées par l'Occident. On observe une dépendance inextricable entre les institutions de conservation de la nature en Afrique (périphérie) et celles de l'Occident (centre) en dépit des efforts déployés pour s’en défaire. Pour Adams, MacShane, Griffiths, Robin, Grove, et MacKenzie (Giraut, Guyot et 
Houssay-Holzschuch, 2003), le débat sur le bien-fondé de l’approche conservationniste souligne ces contradictions et met en cause l'imposition d'un modèle issu d'une représentation européo-centrée de la nature qui s'est élaborée avec les différentes formes de colonisation d’origine européenne et notamment anglo-saxonne. L'idée même de protection peut ainsi être considérée comme une «ingérence écologique ». (Rossi, 2000). Assitou Ndinga (2003) renchérit en disant : « Après l'esclavage et la colonisation, la domination séculaire de l'Afrique Centrale par l'Occident s'est perpétuée dans la post/néocolonisation. L'exploitation brutale des matières premières ainsi que l'expansion du Marché Mondial - la mondialisation - ont dangereusement réduit l'avancée des populations concernées ».

Le deuxième paradigme mobilisé dans cette étude est nommé “réalité d'en bas". Ce paradigme est une remise en question du premier dans la mesure où celui-ci, dans ses pratiques, veut redéfinir les rôles attribués aux institutions de conservation - fortement influencées par la globalisation - de la nature en Afrique et en RDC pour les repenser. Il est donc une émanation de la périphérie qui veut se défaire de l'européocentrisme des institutions de conservation par la recherche d'une nouvelle forme de cohérence en matière de gestion de l'environnement. C'est à juste titre que Chantal Blanc-Pamart et Jean Boutrais (2002) soulignent qu'en Afrique subsaharienne, après le temps des approches sectorielles, zonales et "réparatrices ", s'impose le temps des approches locales, intégrées et participatives.

De ces deux paradigmes, il se dégage un troisième qui est celui de la " réalité d'en face » qui émane de la prise de conscience par l'humanité face à la fragilité planétaire soumise aux modifications diverses, renforcées par les effets de la globalisation. Donc, la logique d'en haut ou du centre c'est la thèse, la réalité d'en bas ou la logique de la périphérie c'est l'antithèse, et la logique d'en face c'est la synthèse, mais qui constitue désormais une nouvelle thèse. En tant que produit du capitalisme périphérique, le PNS reste assujetti à la conception étrangère de la conservation de la nature, qui nécessite actuellement une revisitation, face à l'émergence des exigences du développement durable. Ces contradictions conduisent à des conflits dont le développement provoque des mésententes qui nécessitent à leur tour la mise sur pied d'une nouvelle vision de la conservation. D'une façon dialectique, il s'agit de repenser les institutions concernées par la conservation de la nature. 


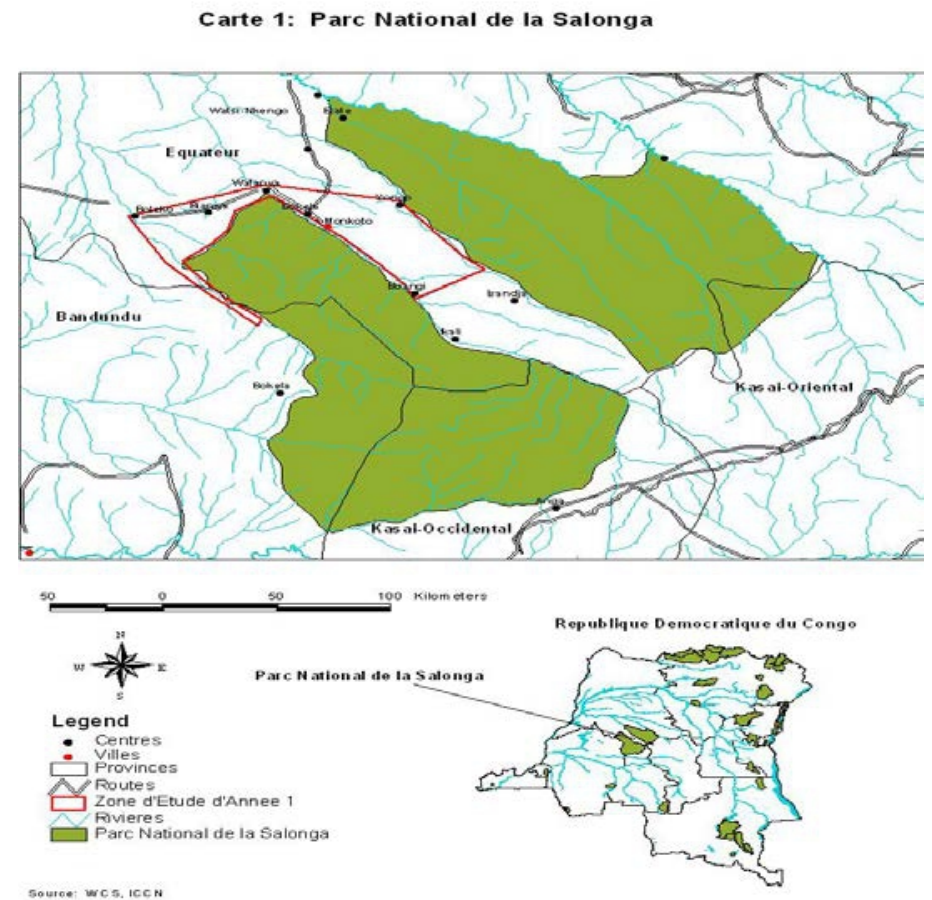

Le parc National de la Salonga compte parmi les sept parcs nationaux de la République Démocratique du Congo (RDC). Il a été créé par l'ordonnance présidentielle $\mathrm{n}^{\circ} 70$ - 318 du 30 novembre 1970 pour la protection de l'immense étendue de forêts tropicales humides et pour la conservation de la faune. Il regorge d'une diversité biologique très importante avec des endémismes. Il est divisé en deux blocs, le bloc nord et le bloc sud, séparés par un corridor habité de plus ou moins quarante kilomètres de large et cent vingt de longueur. Il a une superficie de 36.000 $\mathrm{Km}^{2}$ et se trouve à cheval entre quatre provinces notamment : la province de l'Equateur où est concentrée la plus grande partie dudit parc, la province de Bandundu et les deux Kasaï (oriental et occidental). C’est depuis 1984 que la Salonga a été retenue sur la liste des sites du patrimoine Mondial de l'UNESCO.

\section{Résultats}

Construction des logiques par les acteurs dans la conservation communautaire à la Salonga : logique d'en haut et réalité d'en bas

Depuis quelques années, les mobilisations populaires se multiplient alors que se succèdent les accords internationaux portant notamment sur la couche d'ozone, la désertification, la biodiversité et les gaz à effet de serre. Le dernier en date est la Conférence des parties (Cop 22) tenue à Marrakech au Maroc en décembre 2016. La dégradation de l'environnement n'a pas été 
freinée pour autant, et les aires protégées se dégradent au jour le jour. Le débat lancé ces dernières décennies pour les droits des populations riveraines aux aires protégées, s’est essentiellement focalisé sur les espaces de conservation (parcs nationaux, réserves de faune,...) et la protection des espèces qu'ils contiennent. Paradoxalement, les immenses espaces occupés par les aires protégées, où la question du vécu de ces populations est également cruciale, n’ont suscité, quant à eux, qu'un intérêt limité de la part de la communauté scientifique, en particulier dans le domaine des sciences sociales.

Mc Neely (sd) pense que “ la gestion des aires protégées s’est résumée essentiellement à un travail de surveillance et les populations locales étant considérées comme un problème à la gestion ”. Cette forme de protectionnisme, largement influencé par le colonialisme, qui exploitait les richesses naturelles des pays africains pour la prospérité économique des pays colonisateurs, a encore une grande visibilité au Parc National de la Salonga. Le colonisateur semble avoir oublié que bien des cultures africaines se sont développées au sein de la vie sauvage, qui fait partie de leur héritage dans un sens beaucoup plus réel, et que la chasse et la cueillette de produits sauvages font partie de leur vie quotidienne. Historiquement, en effet, comme le notent Yaa Ntiamoa-baidu et al (sd) “les aires protégées en Afrique ont généralement restreint ou complètement interdit l'accès et l'usage des terres sauvages et de la faune aux communautés locales qui, dans le passé, dépendaient de ces zones pour leur subsistance ”. Cette même logique - dite logique d'en haut - s’est imposée dans la création des parcs nationaux en RDC.

En effet, comme l'écrit Diaw (2002), “ il faudrait d’abord souligner que la politique des aires protégées en Afrique s’est construite sur la dichotomie foncière héritée du colonialisme et du nationalisme foncier. Les néo-protectionnistes radicaux parmi les biologistes de la conservation qualifient d'ailleurs la période des années 60 comme “Le Zénith de la conservation ”. Il était alors plus facile d'imposer les aires protégées sans consulter les populations, en mettant en avant le " capital naturel national " exceptionnel constitué par la faune charismatique africaine ”. Cela s'est fait sans aucun lien avec les institutions ou les valeurs des populations locales.

Cette logique s’affronte à celle dite d'en bas ou de la périphérie, qui veut replacer l'homme au cœur de toute activité et vise à réorganiser ses relations de long terme avec les autres espèces et l'environnement. La plupart des défenseurs des ressources naturelles sont à présent de plus en plus nombreux à se rallier à l'idée que l'homme a toujours influencé la nature et ils sont prêts à reconnaître la nécessité d'impliquer les populations locales dans les projets de conservation de la biodiversité. 
Fort malheureusement, pour les populations riveraines de la Salonga, ce parc est aujourd'hui perçu comme la cause de leur malheur, en dépit des efforts fournis par les acteurs de conservation. Il y a lieu de citer notamment les déplacements forcés de populations, la réduction des superficies agricoles pour la plupart, la quasi-inexistence des rivières pour la pêche, la rigidité des mesures de conservation,.... A cela s'ajoutent les bévues commises par certains agents de l'ICCN, à savoir : le vol, le viol, l'extorsion, les arrestations arbitraires (même en dehors des limites du parc), les intimidations, les taxes illégales, l'incertitude autour des limites du parc,...

Comme on peut s'en rendre compte, toutes les conditions étaient donc réunies pour que l'hostilité sournoise et latente des populations riveraines se manifeste brutalement. Tous les symboles de la conservation ont été attaqués, quelques postes de patrouille (PP) supprimés, notamment à Wafanya, à Bianga et à Boleko. La méfiance, mais également la résistance des populations envers les conservationnistes, s'est accrue, et le braconnage très développé. Il s'est ainsi imposé le constat d'une incapacité foncière à concilier les intérêts contradictoires des acteurs de la conservation (conservationnistes et populations locales) de la Salonga.

Il semble qu'une bonne gestion et une conservation correcte du PNS suppose la compréhension de l'ensemble des facteurs sociaux, politiques, économiques et même culturels et légaux pouvant avoir un impact durable sur ledit parc. C’est la “logique d'en bas”. De ces deux logiques, il en découle une troisième que nous appelons " la logique d'en face ”, qui est le résultat d'une prise de conscience collective de la fragilité de la planète ; fragilité due aux avancées technologiques fondamentales, avec comme conséquences de graves problèmes écologiques. Barbault Robert et al (2002) notent à juste titre que " la biodiversité, fondement même de la vie sur la terre, est aujourd'hui un enjeu majeur, en raison des menaces qui pèsent sur son devenir ”. Il y a donc là un impératif : il faut conserver la biodiversité. Mais comment?

Le véritable défi à la Salonga est de trouver le moyen de préserver les ressources naturelles, la richesse des écosystèmes et la diversité biologique tout en s'assurant qu'elles contribuent pleinement au bien-être de l'ensemble de la population. Nulle part le développement ne s'est construit par la séparation entre les "développés" et leurs ressources naturelles. Mais comment parvenir à une approche consensuelle en regard de tous ces précédents et antécédents, pour des populations déjà en proie à multiples problèmes comme celles de la Salonga ? Il est important de repenser les institutions de conservation, et les adapter à la réalité socioculturelle africaine et congolaise. 
Contraintes pour une conservation citoyenne et une participation communautaire à la Salonga : la gouvernance des aires protégées en question

Nous avons identifié plusieurs contraintes, mais ici nous en discutons les principales.

\section{Inadéquation entre conception et application des mesures de conservation à la Salonga}

L’inadéquation sociale de la politique des aires protégées découle principalement de son incapacité à concilier les intérêts potentiellement et réellement contradictoires des populations locales avec ceux de la conservation. Cet état de choses convoque irrémédiablement la question de la gouvernance qui connaît encore un déficit important. Pour la RD Congo, le véritable défi et de trouver le moyen de préserver les ressources naturelles, la richesse des écosystèmes et la diversité biologique tout en s'assurant qu'elles contribuent pleinement au bien-être de l'ensemble de la population.

Point n'est besoin de rappeler que les projets de conservation en Afrique et en RDC ont vu le jour avec la colonisation. Dans l'élaboration des mesures de conservation, le colonisateur a imposé une forme stricte de protectionnisme qui a été largement influencée par sa politique coloniale d'exploitation des richesses naturelles des pays africains pour la prospérité économique des pays colonisateurs. Selon Yaa Ntimoa et al (sd), “ les leçons tirées de l'histoire nous apprennent que ce type d'approche directive a presque toujours intensifié les conflits à propos de l'utilisation des ressources", lesquels conflits compromettent le développement des populations locales qui auraient dû en être les bénéficiaires.

C’est pourquoi Lloyd Timberlake (1985) affirme que “ généralement, lorsque les pays du Nord pensent à la conservation en Afrique, c'est à la conservation des espèces sauvages qu'ils pensent, aux grandes espèces spectaculaires que la littérature “conservationniste” décrit comme faisant partie de “l'héritage commun à toute l'espèce humaine: éléphants, rhinocéros, lion, gorilles et grandes antilopes ”. Et Diaw (sd) de souligner : “Les conservationnistes considèrent que la biodiversité est un enjeu global mais refusent de mettre leurs richesses nationales sur le même plan. Ainsi, alors qu'ils s'octroient des droits sur les aires protégées des pays en développement, ils n’assument pas en retour leur devoir de solidarité sur les enjeux de répartition de richesses et de liberté de circulation dans les pays industrialisés. Cela revient à dire : “Ouvrez-nous vos parcs, qui sont aussi les nôtres, ceux que Dieu nous a tous offerts en patrimoine, pendant que nous vous fermons nos frontières ".

Les populations riveraines sont considérées comme une menace pour la conservation de la biodiversité à la Salonga. On doit cependant 
reconnaître, actuellement, que des efforts sont fournis par les acteurs en présence (WCS notamment) afin de relever le défi. En même temps, il faut aussi reconnaître les limites auxquelles ces acteurs restent confrontés, notamment la pauvreté des communautés locales, l'enclavement de la contrée, le manque d'accès aux biens et services, l'incertitude des limites, la rigidité des mesures de conservation, ... La mise en œuvre de ces mesures n’a évidemment pas été sans conséquences sur le vécu quotidien des populations locales.

Il y eût des déplacements forcés (depuis 1954 déjà) sans le moindre dédommagement, à en croire nos enquêtés. Ces déplacements opérés sans mesures d'accompagnement (compensations) demeurent la cause principale qui pousse continuellement - bien que sans succès - les populations à des revendications, à des résistances, face aux activités de conservation. Geisler (2003) confirme ce point de vue lorsqu'il affirme : “ Les parcs naturels et les zones protégées dépossèdent les populations de leurs biens et procèdent en des points névralgiques, pour la biodiversité, à un nettoyage des habitants considérés comme une menace pour les parcs et la nature. Il n'est pas rare que ces gens soient pauvres et appauvris encore par l'expulsion. ”

Timberlake (1985) considère que “ les parcs nationaux d'Afrique ont suivi le modèle des parcs nationaux américains, comme ceux de Yellowstone et de Yosemite, dans lesquels aucune activité économique n’est autorisée, pas plus que la chasse, la pêche ou la cueillette. Ces règles ont été établies aux Etats-Unis et formulées par l'Union Internationale pour la conservation de la nature et des Ressources naturelles (UICN) dont le siège est à Gland en Suisse."

Le résultat de l'application de ces règles aux parcs africains est simplement une situation inadéquate dans laquelle des Africains sont engagés, formés et armés pour garder les parcs et pour empêcher des Africains d’y pénétrer, tout cela pour le bénéfice de ceux qui viennent contempler les animaux et des grands hôteliers. Les revenus du tourisme bénéficient aux gouvernants et aux comptes bancaires de certaines personnalités, mais pas du tout aux populations rurales.

Il ressort de ce qui précède que les mesures de conservation en Afrique ne répondent pas aux besoins des sociétés africaines actuelles, mais davantage aux besoins de l'extérieur ; la réalité socio-culturelle de l'Africain et du Congolais n'est que rarement prise en compte. Sournia (1998) reconnaît à cet effet que “ ce refus de prendre en compte ces éléments de la culture, de l'histoire et de la vie quotidienne du continent africain, constitue l'une des aberrations de ces trois dernières décennies en matière d'aménagement du territoire, d'utilisation rationnelle de l'espace et dans la recherche de l'équilibre socio-économique des populations locales des pays africains ". Timberlake (1985) abonde dans ce sens lorsqu'il écrit : 
“L’idée de parcs nationaux, telle qu'elle est actuellement conçue, est une conception étrangère et inacceptable pour la population africaine ”. Selon l'écologiste Kenyan, Walter Lusigi (Timberlake, 1985), “empêcher les Américains des villes de cultiver Yellowstone est une chose, mais empêcher les pasteurs Masai de pénétrer dans la réserve d'Amboseli en est une autre ”. Ou, dans notre cas, empêcher certaines populations du territoire de Monkoto de pénétrer le PNS, c'est les condamner à mourir.

Cette rigidité des mesures de conservation à la Salonga n’offre pas aux populations riveraines l'opportunité de vivre librement. Elles sont obligées de choisir entre les exigences de l'environnement, les contraintes de l'économie et les nécessités de la survie pendant que les ressources naturelles, dont elles dépendent pourtant, leur sont interdites. Pour ces populations - comme nous l'avons déjà dit - le parc de la Salonga reste en grande partie, sinon totalement, la cause des maux dont elles souffrent.

Cette application imposée de théories séduisantes de conservation élaborées sous d'autres cieux et ignorant tout du contexte local, provoque une certaine "gauchisation » des réactions dans le chef des populations, et fait appel à de nouvelles formes de résistance face aux activités de conservation. Ces résistances prennent des formes à la fois spontanées, mais organisées, et se traduisent par le sabotage, la rancœur, la méfiance, ... et ce, en dépit des interventions des acteurs de la conservation. Par cette méthode, des projets de développement mal ajustés aux besoins de la population sont imposées. C’est pourquoi Bertin et al (2006) pensent que “ l'un des paradoxes du développement repose sur l'articulation de la décision locale avec le rôle de l'agent de développement exogène ”. Cette approche à la Salonga reste la source de nombreux conflits relatifs notamment aux limites du parc, aux déplacements forcés, etc.

\section{Le déplacement des populations en question à la Salonga : discours et contre-discours}

Il s’agit ici des déplacements imposés aux populations actuellement riveraines au PNS. Notre attention s'est surtout focalisée sur les populations résidant les groupements ci-après : Wafanya, Nkole Lokolo (situés dans le secteur de Bianga sur l'axe routier Wafanya - Boleko dans le bloc Sud du PNS) de Bolengangele, Imomampako, Isaka Entow, Emengeye, Bosongo, Ilanga, MPenge Kaboko, Ndomba Sud, Booli, Boono Boene et Nongelokwa (situés en grande partie dans le corridor séparant les deux blocs du PNS et en partie au bloc Nord).

Ces populations, après avoir construit et consolidé leur culture, après avoir acquis un sentiment d'appartenance et de propriété, etc., sont actuellement victimes d'un déplacement qui rappelle l'époque de l'esclavage, avec l'impossibilité de posséder et de conserver des biens, la 
souffrance et les mauvais traitements infligés par ceux qui sont détenteurs (propriétaires) des terres et des rivières dans la zone de réinstallation. Tout cela fait partie de la “ mémoire collective ” comme l’a écrit Rosero (2003).

Les déplacements auraient été imposés à ces populations dans le but de conserver la biodiversité pour laquelle les populations locales constituaient - selon le colonisateur - une menace. Elles étaient disséminées dans les deux blocs. Les unes dans le Sud, au-delà de la rivière Losoy (Budjaosoy qui signifie au-delà de la Losoy) et les autres - surtout celles vivant actuellement dans ce vaste corridor - entre les rivières Salonga et Iyenge. Disons, dès à présent, que le discours ici c'est l'intention (puis la décision) du colonisateur, tandis que le contre-discours est constitué par les revendications foncières de certaines populations locales, et la résistance d'autres. Selon ces populations, les déplacements auraient commencé à partir de 1941 ; pour certaines d'entre-elles, cela s'est passé en une fois, pour d'autres en deux fois. Celles qui vivaient dans le bloc Sud par exemple, seraient parties de la forêt jusqu'au long de la route où elles se situent actuellement. Celles qui résidaient dans le bloc Nord auraient quitté d'abord leur première résidence située entre la Salonga et Iyenge, puis seraient quelques années peu après - parties de là pour la grand-route car - aux yeux du colonisateur - elles constituaient une menace pour la réserve qui était déjà en projet.

Les discours du colonisateur n’ont pas été francs. Il aurait caché le motif de déplacement aux populations en disant que toutes les populations qui logeaient encore dans la réserve, devaient rester le long de la route pour faciliter les opérations administratives du colonisateur, tel le recensement, l'assistance... Il ne s'agit pas de contester que ces déplacements aient été motivés par l’intention de conserver la biodiversité. Albert (2003) qualifie ces déplacements “d'expropriation écologique”. Celle-ci a fait de ces déplacés des “ réfugiés pour cause de conservation de la nature ”.

Mais l'une des caractéristiques de l’exécution de ces grands projets de développement est le déplacement forcé des populations et la pauvreté qui l'accompagne, car souvent les déplacés se voient obligés d'abandonner, non seulement leurs biens, mais aussi leurs lieux de résidence, leurs réseaux sociaux ainsi que leur contrôle et accès aux biens. Michael (2003) le souligne bien en ces termes: “Les populations déplacées sont souvent exposées aux dangers suivants: perte de la terre, perte d'emploi, perte de logement, marginalisation, accroissement de la morbidité et de la mortalité, détérioration des possibilités d'éducation, insécurité alimentaire, perte d'accès aux biens collectifs et la désagrégation sociale ”. C’est pour cette raison que, sur le plan économique et culturel, les déplacements forcés sont l'équivalent d'un tremblement de terre majeur anéantissant les systèmes de 
production et le réseau des relations sociales, affaiblissant les identités et jetant les sinistrés dans la spirale de l'appauvrissement.

Les initiateurs du projet de la conservation à la Salonga n’ont pas anticipé les dégâts humains, réels ou potentiels, que les déplacements forcés allaient entraîner. Pourtant, il peut y avoir des affinités entre la conservation et le développement comme le rappelle Geisler (2003) pour qui “ la création des zones protégées constitue souvent une stratégie de développement en soi et que ce n'est que lorsque ce point sera acquis que le phénomène des réfugiés pour cause d'opération de conservation pourra se comprendre ". Mais pour les déplacés de la Salonga, 35 ans après sa création, les effets escomptés de cette affinité entre la conservation et le développement ne sont pas au rendez-vous. Le parc constitue, pour eux, un obstacle qui, non seulement les appauvrit en les dépossédant, mais aussi les avilit en les empêchant de satisfaire correctement leurs besoins vitaux.

\section{Nécessaires désillusions pour une conservation citoyenne en Afrique et en RDC}

L’engagement de repenser les rôles des institutions de conservation de la nature est d'abord, comme l'écrivent Ekili et Amisi (2004) à propos de la reconstitution de la réalité africaine, “ un travail d’écriture et de réécriture ayant une double vocation de mystification et de démystification ”. Il en faut pour le Congo dans le secteur de la conservation de la nature. Les désillusions sont nécessaires pour mettre au jour les faiblesses qui soustendent la logique de conservation héritée de la colonisation, laquelle s’affiche comme totalement coupée des réalités des sociétés africaines. Leurs échecs successifs, tragiquement ressentis par les communautés locales, témoignent de la dépendance dans laquelle les Africains ont été longtemps plongés par la colonisation. C’est à ce titre que Mudimbe (2004) écrit : “ La relecture africaine exprime l'exigence, dans une perspective critique, mais aussi fondatrice, d'une réappropriation d'un discours africain authentique ”.

Comme signalé plus haut, notre préoccupation n’est pas ici de rejeter en bloc les mesures de conservation et leurs institutions. Nous tenons plutôt à redéfinir leurs rôles qui, dans “ l'agir social ”, traduisent plus la réalité occidentale que celle africaine et congolaise. Or, le développement, comme l'indique l'Abbé Paul Houée (1972) dans une distinction qui a fait école, doit être une praxis puisque la transformation du social produit des effets sur les acteurs qui, eux-mêmes, contribuent à sa transformation.

Quelle attitude adopter face à cette situation, sinon la recherche d'un nouveau paradigme de la cohérence en matière de gestion de l'environnement? Il faut donc trouver un nouveau paradigme capable de susciter de nouvelles réflexions pour une nouvelle sociologie, à la fois socialement utile et théoriquement novatrice. On se trouve donc en présence 
d'une contre-recherche sur le développement à partir d'une conservation citoyenne qui consiste, non seulement à dépasser les anciennes théories européocentriques, extraverties du développement, mais aussi à procéder à leur relecture critique en vue d'une connaissance débarrassée des préjugés, et prenant en compte la réalité socio-culturelle de l'Africain et du Congolais. Perroux (Bertin et al, 2006) soutient à cet effet que “ les valeurs culturelles jouent un rôle fondamental dans la croissance économique qui n’est rien d'autre qu'un moyen ; les valeurs culturelles sont à la base des motivations qui freinent ou accélèrent la croissance et de légitimation des objectifs de la croissance ".

Le nouveau paradigme de cohérence dans le domaine de la conservation devra se défaire de l'impérialisme culturel de l'Occident en considérant la réalité socio-culturelle de l'Africain comme un paradigme pour rapprocher la théorie existante du vécu des communautés locales ; la culture étant définie comme " un élément fondamental de la vie de chaque individu et de chaque communauté, le développement, dont l'homme est la finalité, possède une dimension culturelle essentielle' (Conférence Mondiale sur les Politiques Culturelles à Mexico en 1982). La science de la conservation et sa pratique doivent répondre à l'orientation utilitaire de la conservation en Afrique et au Congo, en tenant compte autant de la vie sociale des communautés locales que de principaux concepts opératoires (la conservation, la culture, le développement local et durable, l’environnement...) indispensables à la sociologie environnementale.

Dans cette nouvelle dynamique, il faudra donc protéger "les différentes entités culturelles” pour éviter, comme écrit Mwabila (2004), présentant l'ouvrage de Bongeli, “ de naviguer à vue dans le domaine de l'agir social, de peur de déboucher sur les malheureux atterrissages forcés ”. Fort malheureusement, ces atterrissages forcés ont déjà eu lieu dans le domaine de la conservation au Parc National de la Salonga. Il convient donc plutôt, à la lumière de ce nouveau paradigme, de préparer un nouveau décollage en douceur: celui de la conservation citoyenne qui devra tenir compte, non seulement des intérêts des populations locales, mais aussi et surtout de leur culture historiquement établie. Cette citoyenneté suppose, comme le soutient Bongeli (2004), présentant l’ouvrage de Mukoka, “ une implication positive des citoyens dans les affaires publiques " : " que chacun s'acquitte de ses devoirs et obligations et assure la jouissance par les concitoyens de leurs droits, l'esprit de créativité et la culture du travail qui font des citoyens des agents de développement et, enfin, le sens des devoirs collectifs au sein de la communauté”.

Considérons par exemple cette mesure qui interdit strictement aux populations riveraines de «s'aventurer » au sein du Parc National de la Salonga sous peine d'arrestation et d'amendes, alors que, dans la pratique, il 
y en a parmi elles qui ne sont séparées de la réserve que de quelques mètres. Comment peuvent-elles vivre s’il faut les priver de ce qui fonde leur existence dans leur environnement? Ceci fait apparaître clairement l'insouciance des acteurs de la conservation à l'égard des communautés vivant aux environs du parc, alors qu'une conservation durable de ce parc passe nécessairement par elles eu égard à cette nouvelle dynamique. Le souci des concepteurs de ce parc ne visait qu'à protéger la grande forêt tropicale humide et les espèces endémiques qui s’y trouvent. Nous observons ici l'extraversion de toutes ces mesures de conservation, qui ne rencontrent pas les attentes et les besoins des communautés locales, mais plutôt ceux de l'extérieur.

Si la mise en œuvre de toutes ces mesures, et même les théories en matière de développement avancées par les organisations internationales, se sont révélées inefficaces dans le contexte africain, c’est qu'elles sont toutes issues de l'Occident. Elles ont été conçues en Occident sans tenir compte des réalités africaines. Elles ne répondent pas à la culture africaine, et, par conséquent, ne peuvent pas contribuer à son développement. Mwabila (2000) écrit à juste titre que “ face à la faillite des stratégies du développement proposées jusqu'ici aux pays du tiers monde, particulièrement à l'Afrique, la nécessité se fait sentir de repenser la théorie, les pratiques et les stratégies du développement". Et Sournia (1998) de renchérir, qu'un certain nombre d'observateurs estiment que les politiques de conservation sont, à l'origine, issues des sociétés industrielles et urbaines du monde occidental. Ils en déduisent que les pratiques qui en découlent ne peuvent qu'occulter les incidences locales (1998). Vu sous cet angle, poursuit l'auteur, “ la création des espaces protégés en Afrique s’apparente au néo-colonialisme, bien que ces choix correspondent également à une forme de protection des intérêts locaux".

Il est à remarquer que les Etats africains “indépendants" ne remettent pas en cause cette situation, notamment parce que les parcs nationaux sont toujours considérés comme une source de devises étrangères en raison d'un tourisme en développement constant. Cette situation nécessite l'introduction d'une dimension nouvelle dans la conception des aires protégées en Afrique qui, dépassant leur rôle de conservatoires de l'environnement, doivent contribuer nécessairement au "développement économique ”, en créant des richesses sur lesquelles les populations locales auront des droits réels. Il n’est donc pas réconfortant aujourd’hui de constater que les concepteurs et les initiateurs des aires protégées n’intègrent pas encore cette préoccupation. Ils ne pensent même pas à la nécessité de s'accorder aux réalités locales et à celles de ce siècle, à un moment où l'harmonie des relations entre l'homme et son milieu est mise à mal. Une 
telle attitude risque d'hypothéquer l'avenir en ignorant les besoins fondamentaux de ceux qui vivent sur les territoires voués à la conservation.

Pour rappel, le développement durable a été défini dès 1987 par l'ONU (1987) comme “ un développement qui tient compte des besoins actuels sans compromettre les besoins des générations futures ”. Dans la pratique, comment les communautés locales à la Salonga peuvent-elles garantir les besoins des générations futures alors qu’elles répondent déjà difficilement aux leurs actuels? Il existe à la Salonga des communautés qui ne sont séparées de la réserve que d'un kilomètre, voire moins d'un kilomètre. Cette position géographique les oppose régulièrement aux gardes du parc et favorise de nombreux conflits. Pour ces communautés (situées en grande partie dans le secteur administratif de Monkoto), le parc ne constitue rien d'autre qu'un problème. Il est impossible d'imaginer qu'elles puissent s'impliquer dans la conservation communautaire alors qu'elles sont incapables de résoudre leur problème de survie du fait de la rigidité des mesures de conservation pesant sur elles et sur l'espace déclaré patrimoine mondial de l'humanité.

En toute évidence, il est même paradoxal de constater que l'UNESCO ne propose pas de mesures de mise en œuvre des définitions qu'elle-même élabore sur le développement des communautés. L’UNESCO a financé des enquêtes socio-économiques détaillées sur les populations riveraines au Parc National de la Salonga en vue d'un projet de conservation communautaire (nous y avons participé). D’abord, il faut avouer que ce projet était conçu indépendamment de besoins de la base, donc sans lien essentiel avec le vécu des populations. La mise en œuvre de ce projet n’a fait que consacrer le renforcement de l'extraversion et de l'eurocentrisme tant décriés par nombre d'observateurs. Entretemps, nous savions, en tant qu'enquêteurs, que ces populations ne pourraient vivre que des ressources tirées de leur environnement immédiat, mais qui, paradoxalement, leur sont interdites parce que situées dans la réserve.

Il est également paradoxal que l'Institut Congolais pour la Conservation de la Nature (ICCN) puisse prétendre conserver une si grande forêt $\left(36.000 \mathrm{~km}^{2}\right)$ avec des moyens tout aussi limités. Le dispositif sécuritaire est très précaire. Il existe à la Salonga six stations, chacune d’elles étant constituée de plus ou moins de trois postes de patrouille. Mais ces postes de patrouille sont sous-équipés, avec un personnel lui-même vieillissant. Les braconniers étant toujours mieux équipés qu’eux, ils les délocalisent régulièrement et opèrent dans une impunité jamais égalée. Si l’on n’y prend pas garde, les générations futures risquent donc de connaître d'énormes difficultés en ressources naturelles. Les institutions de conservation ont un grand défi à relever pour gagner le pari d'un 
développement des communautés locales passant par une bonne mise en œuvre des principes de conservation.

Ainsi, en vue d'atteindre cette conservation citoyenne tant souhaitée en Afrique et au Congo - Kinshasa, c'est-à-dire une conservation qui tienne compte des organisations humaines établies dans des zones sensibles, le nouveau paradigme doit aussi intégrer la participation communautaire en amont et en aval. En d'autres termes, il faudrait amener les communautés locales à une participation effective et active. En amont, il faudrait les associer à la conception même des projets dont elles sont souvent théoriquement les bénéficiaires pour éviter toute inadéquation sociale. En aval, il convient de les faire participer non seulement à l'exécution du projet, mais aussi à la prise de décisions et au suivi, voire l'évaluation. De la sorte, ces populations seraient prêtes à soutenir la conservation ; en revanche, les institutions de conservation de la nature sortiraient victorieuses en suscitant le développement des communautés locales.

En plus de cette participation communautaire, le nouveau paradigme de cohérence devra intégrer dans sa démarche la promotion de l'éducation mésologique et même du tourisme. Bien que riveraines au Parc national de la Salonga, bon nombre de populations ne connaissent pas encore l'importance de celui-ci tant sur le plan écologique qu'économique et politique. Parlant des aires protégées d’Afrique francophone, Sournia (1998) déclare que “ les questions politiques ne furent jamais absentes des grands problèmes de conservation,... la nature sauvage et les aires protégées sont parfois utilisées comme un moyen d'exalter la fierté et les sentiments nationalistes ”. Les populations locales à la Salonga devront être sensibilisées aux acquis du parc et ses incidences socio-économiques, politiques et même culturelles.

En plus de cette sociologie de l'environnement, la sociologie de la conservation, selon l'heureuse expression de Sournia (1998), est envisageable à l'échelle de la Salonga en RDC et de toute l'Afrique. Elle devra avoir pour objectif d’identifier les facteurs gouvernant l'évolution des sociétés humaines, et particulièrement les organisations humaines riveraines aux aires protégées, d'appréhender les modes de vie des sociétés africaines actuelles, leurs attitudes par rapport aux ressources naturelles et les usages qu'elles en font. Il lui faudra savoir analyser les modes d'utilisation de l'espace, les systèmes de production, leur impact sur la biodiversité en général, et comprendre les logiques d’invention des différents groupes sociaux et les interactions entre ces groupes, pour pouvoir, en cas d'usages conflictuels, identifier les éléments sur lesquels les intérêts des différents utilisateurs convergent et, à partir de là, amorcer un processus de négociation dans une approche patrimoniale. 


\section{Conclusion}

Parlant de son livre Odeur du père, Mudimbe (2000) cite avec pertinence B. Verhaegen (1977) qui affirme que “l 'université africaine, même si elle est localisée en Afrique, n’est pas africaine, elle le deviendra le jour où elle se souciera de contribuer le mieux possible à connaître et à résoudre les contradictions des sociétés africaines, et prendra la place qui lui incombe dans la formation des places sociales nouvelles dans une Afrique confrontée au défi de son développement et son adaptation au monde moderne".

A la lumière de cette pensée, nous considérons aussi qu'un parc africain, même s’il est localisé en Afrique, n’est pas africain, surtout lorsqu’il est déclaré patrimoine international de l'Unesco. Il le deviendra le jour où les acteurs de la conservation parviendront à intégrer dans leur politique de conservation, les contraintes socio-économiques, politiques et culturelles des populations locales en vue d'une conservation citoyenne. Le besoin est réel de se défaire de cette extraversion des institutions de conservation et de leur européocentrisme, en mettant en œuvre un nouveau paradigme, capable de susciter de nouvelles réflexions pour une nouvelle sociologie à la fois socialement utile et théoriquement novatrice.

Terminons ainsi avec Provent et Ravignant (1977) en soulignant ceci : «Si l'on voulait être efficace, il faudrait d'abord admettre qu'il n'y a pas de développement sinon par tous et pour tous. Qu'il n’y a pas de respect possible de l'environnement tant qu'il subsiste dans une région des zones de sous -développement...». Autrement dit, pour atteindre une Afrique positive dans la conservation de la nature, les acteurs sociaux concernés devront être préoccupés par la prospérité des populations locales ; faute de quoi, la conservation citoyenne tant souhaitée demeurera un leurre.

\section{References:}

1. Amuri Misako, F. (2012). La symbolique de la légitimation de la violence milicienne en Afrique. Continuités et réinventions du messianisme nationaliste chez les Mä̈-Maï du Maniema au CongoKinshasa, Sarrebruck, Editions universitaires européennes.

2. Barbault, R. et al. (2002). Johannesburg sommet mondial $d u$ développement durable 2002. Quels enjeux ? Quelle contribution des scientifiques ? Ministère des affaires étrangères, France.

3. Bertin, G., et al. (2006). Développement local et intervention sociale, Paris, L'Harmattan.

4. Bongeli Yeikelo ya Ato, E. (2004). «Discours et pratiques du développement en RDC: regards pathologiques de Mukoka Nsenda », Analyses sociales, volume IX, numéro unique, janvier décembre, (pp. 179-191) 
5. Henri, B. (2006). Module de formation en gestion participative des ressources forestières, Bureau National du programme WWF en RDC, décembre.

6. Ekili Tabu, G. et Amisi Awezai, B. (2004). «Pour une nouvelle lecture des problèmes de la RDC: nécessaires désillusions », Analyses sociales, volume IX, numéro unique, janvier-décembre, (pp.72-80).

7. Escobar, A. (2003). “ Déplacement, développement et modernité en Colombie du Pacifique", Revue internationale des sciences Sociales, $\mathrm{n}^{\circ} 175$, Mars, (pp. 171 - 182).

8. Geisler, C. (2003). “ Les expulsés des jardins d’Eden : un nouveau problème ", Revue internationale des Sciences Sociales, $\mathrm{n}^{\circ} 175$, mars, (pp. 73-83).

9. Giraut, F., Guyot, S. et Houssay-Holzschuch, M. (2003). Les aires protégées dans les recompositions territoriales africaines, Ve congrès mondial des parcs tenu à Durban-Afrique du Sud.

10. Houée, P. (1972). Les étapes du développement rural, Paris, Ed Ouvrières.

11. Kambayi Bwatshia. (2004) “L’odeur du père de V.Y. Mudimbe ou stratégie pour rompre la dépendance”, Analyses sociales, Volume IX, numéro unique, janvier - décembre, (pp. 157-166).

12. Lourau, R. (1973). L'analyse institutionnelle, Paris, Ed de Minuit.

13. Michael, M.-C, (2003). "Pour une nouvelle économie de la réinstallation : critique sociologique du principe de compensation ”, Revue Internationale des sciences sociales, $\mathrm{n}^{\circ} 175$, mars, (pp. $39-$ 48).

14. Mwabila Malela C. (2000). "Impact de la globalisation sur la culture de la pauvreté", in Firouzeh Nahavandi, Globalisation et néolibéralisme dans le tiers-monde, Paris, L’Harmattan, (pp. 172178).

15. Mwabila, Malela, C. (2004). “Sociologie et sociologue africains. Pour une recherche citoyenne au Congo-Kinshasa de Bongeli ”, Analyses sociales, Volume IX, numéro unique, janvier - décembre, (pp. 172 - 178).

16. Ndinga, A. (2003). Domination et résistance de l'Afrique centrale. Changement et enjeux, Paris, L’Harmattan.

17. Provent, A. et Ravignan, F. (De) (1977), Le Nouvel ordre de la faim, révolutions paysannes, éd. Seuil, Paris.

18. Rossi, G. (2000). L’ingérence écologique. Environnement et développement rural du Nord au Sud, Paris, CNRS Editions.

19. Sournia. G. (dir.). (1998). Les aires protégées d'Afrique francophone, Paris, Jean-Pierre de Monza. 
20. Timberlake, L. (1985). L'Afrique en crise. La Banqueroute de l'environnement, Paris, L'Harmattan.

21. UN. (1987). Rapport pour les Nations Unies.

22. Verhaegen, B. (1977). L'impérialisme technologique et bourgeoisie nationale au Zaïre ", Connaissances du Tiers-monde, 10-17.

23. Yaa Ntiamoa Baidu et al. (s. d.). Principes et Pratiques. Observations du Personnel des projets de conservation en Afrique, Biodiversity support Program, Washington DC. 